\title{
Checkpoint inhibitor-induced fulminant myocarditis, complete atrioventricular block and myasthenia gravis - a case report
}

\author{
Antonio Portolés Hernández ${ }^{1}$, Mariola Blanco Clemente ${ }^{2}$, Daniel Escribano García ${ }^{1}$, \\ Rocío Velasco Calvo ${ }^{3}$, Beatriz Núñez García ${ }^{2}$, Juan Francisco Oteo Domínguez ${ }^{1}$, Clara Salas Antón ${ }^{4}$, \\ Miriam Méndez García ${ }^{2}$, Javier Segovia Cubero ${ }^{1,5}$, Fernando Domínguez ${ }^{1,5}$ \\ ${ }^{1}$ Department of Cardiology, Hospital Universitario Puerta de Hierro, Madrid, Spain; ${ }^{2}$ Department of Oncology, Hospital Universitario Puerta de \\ Hierro, Madrid, Spain; ${ }^{3}$ Department of Neurology, Hospital Universitario Puerta de Hierro, Madrid, Spain; ${ }^{4}$ Department of Pathology, Hospital \\ Universitario Puerta de Hierro, Madrid, Spain; ${ }^{5}$ CIBERCV, Instituto de Salud Carlos III, Madrid, Spain \\ Correspondence to: Dr. Fernando Domínguez, MD, PhD. Heart Failure and Inherited Cardiac Diseases Unit, Department of Cardiology, Hospital \\ Universitario Puerta de Hierro, Manuel de Falla 1, ZC 28222, Majadahonda, Madrid, Spain. Email: fdominguezrodriguez@gmail.com.
}

\begin{abstract}
Immune checkpoint inhibitors (ICIs) have revolutionized cancer therapy over the last decade. Pembrolizumab, a humanized monoclonal IgG4 antibody, binds to the programmed death 1 (PD-1) receptor, blocking its interaction with programmed death-ligand 1 (PD-L1) and thereby increasing the antitumor activity of the host immune system. These drugs are associated with immune-mediated side effects that can be life threatening, and myocarditis is among the most serious events. We present a 48-year-old woman with a history of progressive thymoma who developed complete atrioventricular block associated with fulminant myocarditis and myasthenia gravis 2 weeks after starting treatment with pembrolizumab. She had also presented a couple of days before to the emergency department due to dyspnea that was related to pleural effusion. Electrocardiogram (ECG) and echocardiogram were unremarkable, but she had very mildly increased troponin levels that were attributed to acute respiratory compromise, so she was discharged after successful thoracentesis. Despite aggressive treatment combination of high-dose corticosteroids, immunosuppressive agents and anti-thymocyte globulin, the disease rapidly progressed to the fatal outcome. This report remarks on the importance of rapid consideration of ICI-induced myocarditis even if cardiac biomarkers are slightly elevated, as a mild presentation can go unnoticed and progress to a severe case. Therefore, a high index of suspicion is warranted in these patients and cardiac imaging techniques such as magnetic resonance could have a role diagnosing incipient cardiac inflammation.
\end{abstract}

Keywords: Immune checkpoint inhibitors (ICIs); myocarditis; early diagnosis; immunosuppression

Submitted Mar 07, 2021. Accepted for publication Jun 20, 2021.

doi: $10.21037 / \mathrm{cdt}-21-147$

View this article at: https://dx.doi.org/10.21037/cdt-21-147

\section{Introduction}

Immune checkpoint inhibitors (ICIs) have emerged as promising treatment options for patients with advanced staged cancer (1). These monoclonal antibodies help to restore antitumor immunity by targeting negative regulation receptors and promoting tumor cell death. These targets include the cytotoxic $\mathrm{T}$ lymphocyte antigen
4 (CTLA-4), programmed death 1 (PD-1) or its ligand, programmed death-ligand 1 (PD-L1). There are currently seven FDA-approved ICIs for cancer therapy, which include ipilimumab (anti-CTLA-4), nivolumab, pembrolizumab, cemiplimab (anti-PD-1), avelumab, atezolizumab and durvalumab (anti-PD-L1). However, these drugs may induce inflammatory side effects as a result of the increased immune system activity, which are generally termed as

\footnotetext{
^ ORCID: 0000-0001-8408-363X.
} 


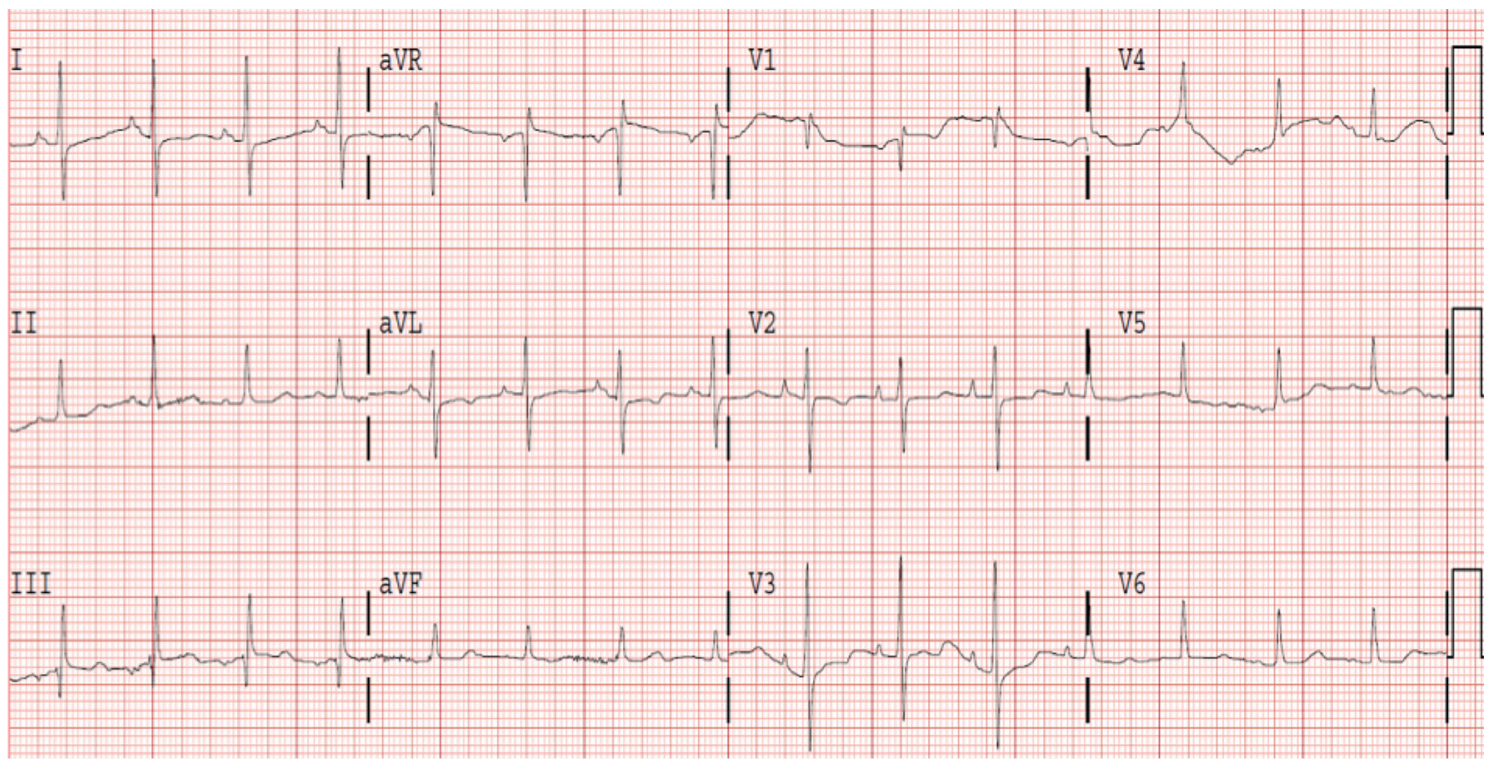

Figure 1 First ECG. ECG, electrocardiogram.

immune-related adverse events (irAEs) (2). Cutaneous, gastrointestinal, hepatic and respiratory are among the most commonly observed irAEs, while others such as hematological or cardiac are infrequent. Common cardiac irAEs include atypical chest pain, arrhythmias and new onset heart failure. Among cardiac irAEs, myocarditis is rare but feared the most as the clinical course has often been described as fulminant $(3,4)$. We present the following case in accordance with the CARE reporting checklist (available at https://dx.doi.org/10.21037/cdt-21-147).

\section{Case presentation}

We present a 48-year-old woman with a history of stage IV-A thymoma (WHO Type B3) diagnosed in January 2013. She was treated with four cycles of cisplatin/ doxorubicin/cyclophosphamide (CAP), followed by surgical resection of the primary tumor and mediastinal pleural nodules (no clear margins) and concurrent radiation. Between March 2015 and September 2020, PET scans showed progressive disease with lung, pleural and bone metastases despite recurrent surgical resection, stereotactic body radiation therapy and four additional cycles of CAP (total dose of doxorubicin: $394 \mathrm{mg} / \mathrm{m}^{2}$ ). The patient presented high PD-L1 immunohistochemistry expression $(70 \%)$ and second line systemic treatment with pembrolizumab ( $2 \mathrm{mg} / \mathrm{kg}$ every 3 weeks) was started in October 2020. She had no history of previous autoimmune or cardiovascular diseases.

The patient presented to the emergency department 10 days after the first dose of pembrolizumab with progressive shortness of breath that had gradually worsened during the previous days. Chest radiography showed severe right pleural effusion and laboratory test showed mild elevation of highly sensitive troponin I (207 pg/L; reference: $<48$ pg/L). The electrocardiogram (ECG) and transthoracic echocardiogram performed were normal (Figure 1). Repeated troponin levels showed no further increase and the patient was discharged after successful thoracentesis. Three days later she was readmitted to the hospital presenting with recurrent dyspnea. Blood pressure at the emergency room was $110 / 60 \mathrm{mmHg}$, heart rate 40 beats/min, respiratory rate 30 breaths/min and oxygen saturation $85 \%$. Chest radiography showed reappearance of pleural effusion (Figure 2) and ECG showed complete atrioventricular block with QRS duration of $130 \mathrm{~ms}$, right bundle branch pattern and an escape rhythm with a rate of 40 beats $/ \mathrm{min}$ (Figure 3).

Laboratory test results on readmission were significant for the following: elevated $\mathrm{N}$-terminal pro-brain natriuretic peptide (NT-proBNP): 1,448 pg/mL (reference: $<125 \mathrm{pg} / \mathrm{mL}$ ), elevated high sensitive troponin I: 4,776 pg/L, leukocytosis with neutrophilia, elevated C-reactive protein $(63 \mathrm{mg} / \mathrm{L}$, reference: $<10 \mathrm{mg} / \mathrm{L})$, creatine kinase $(2,430 \mathrm{U} / \mathrm{L}$, reference: $24-170 \mathrm{U} / \mathrm{L}$ ) and mild transaminitis (aspartate 
transaminase, $179 \mathrm{U} / \mathrm{L}$; and alanine transaminase, $68 \mathrm{U} / \mathrm{L}$ ). Pleural effusion was drained again to palliate symptoms, and the analysis showed a predominantly lymphocytic exudate with negative cytology. Transthoracic echocardiogram revealed new-onset mildly depressed left ventricular (LV) ejection fraction of $45 \%$ to $50 \%$ with neither ventricular hypertrophy nor segmental wall motion abnormalities. Coronary angiogram showed normal coronary arteries. LV endomyocardial biopsy revealed lymphocytic infiltration (CD3+ and CD20-) of the myocardium and interstitial space with mild muscle fiber injury, consistent with acute lymphocytic myocarditis. Some eosinophils were additionally observed (Figure 4).

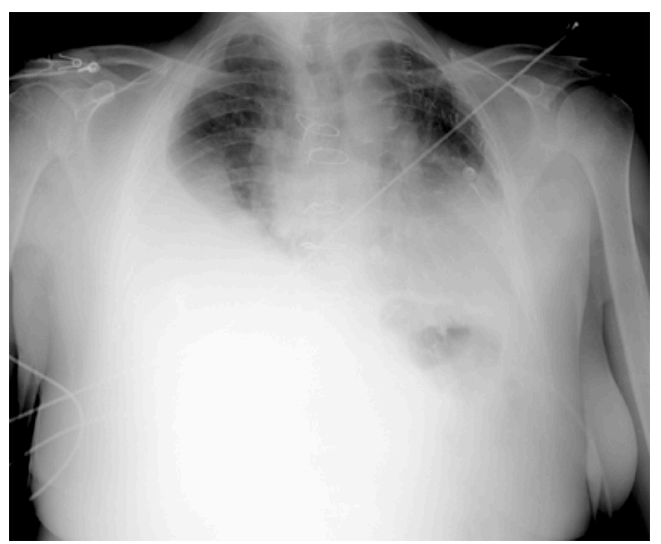

Figure 2 Admission chest radiography showing significant right pleural effusion.
The patient was admitted to the intensive coronary care unit, placed on intravenous isoproterenol drip and received the first methylprednisolone dose $(2 \mathrm{mg} / \mathrm{kg})$ within 2 hours from the first time immune-mediated myocarditis was suspected. Given that atrioventricular block persisted and both cardiac biomarkers and C-reactive protein increased (troponin peak reached: $13,343 \mathrm{pg} / \mathrm{L}$ ), a single Infliximab dose $(5 \mathrm{mg} / \mathrm{kg})$ was administered, a temporary pacemaker implanted and methylprednisolone dosage escalated $(1 \mathrm{~g}$ per day, intravenously, for 5 days), and subsequently continued with previous dose $(2 \mathrm{mg} / \mathrm{kg})$. Despite this treatment, conduction disturbances and clinical status worsened (NT-proBNP peak reached: 8,100 pg/mL). Therefore, a dual-chamber pacemaker was implanted. Subsequent echocardiography examinations showed biventricular failure along with signs and symptoms of congestive heart failure (LV ejection fraction decreased to $25-30 \%$ ). Cardiac rhythm became unstable, showing a rapid and polymorphic ventricular rhythm (rate 90-100 beats/min) (Figure 5A) managed with faster ventricular pacing (Figure $5 B$ ) and intravenous amiodarone. Noradrenaline and dobutamine were started and two doses of intravenous anti-thymocyte globulin were administered due to unresponsive hypotension and oliguric renal failure. The latest echocardiogram showed very severe biventricular dysfunction (LV ejection fraction: $10 \%)$.

Along these days, the patient also developed mild bilateral ptosis and blurred vision. Testing for acetylcholine receptor antibodies was positive and pyridostigmine was

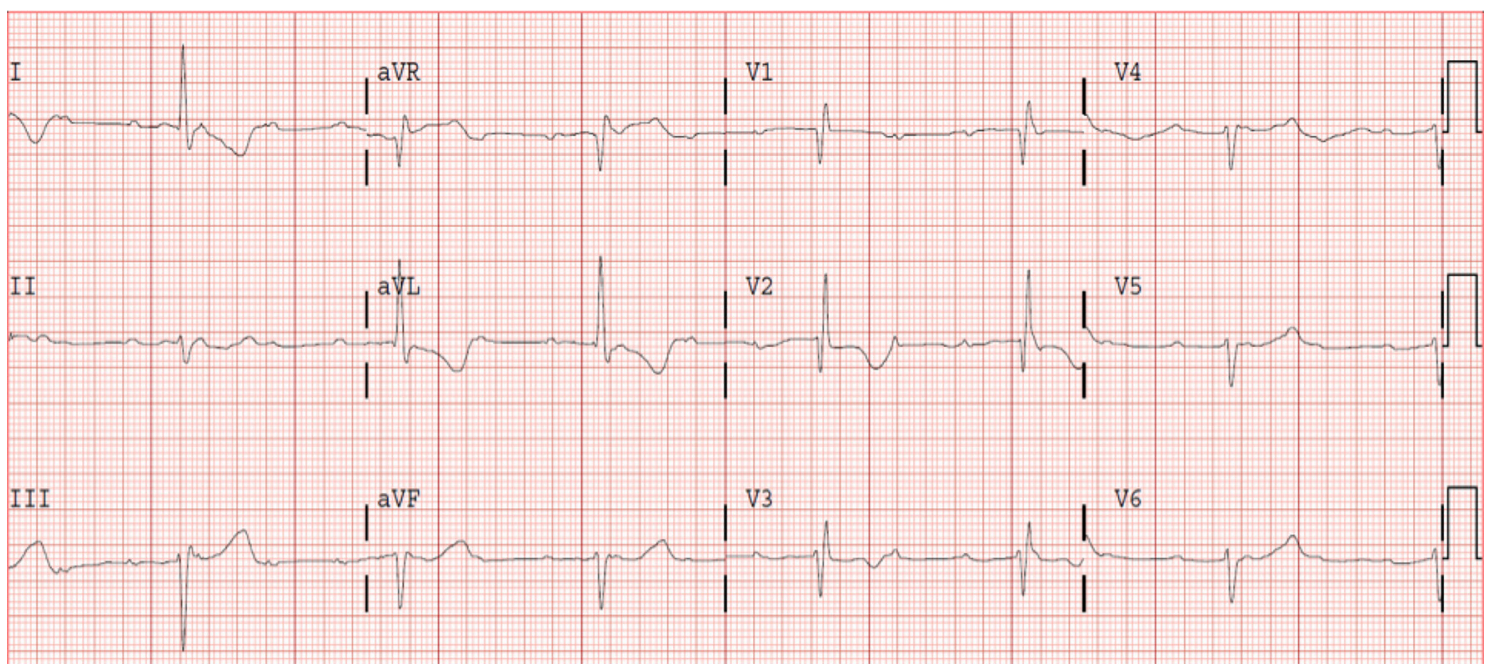

Figure 3 Admission ECG shows complete atrioventricular block with right bundle branch pattern and an escape rhythm of 40 beats/min. ECG, electrocardiogram. 

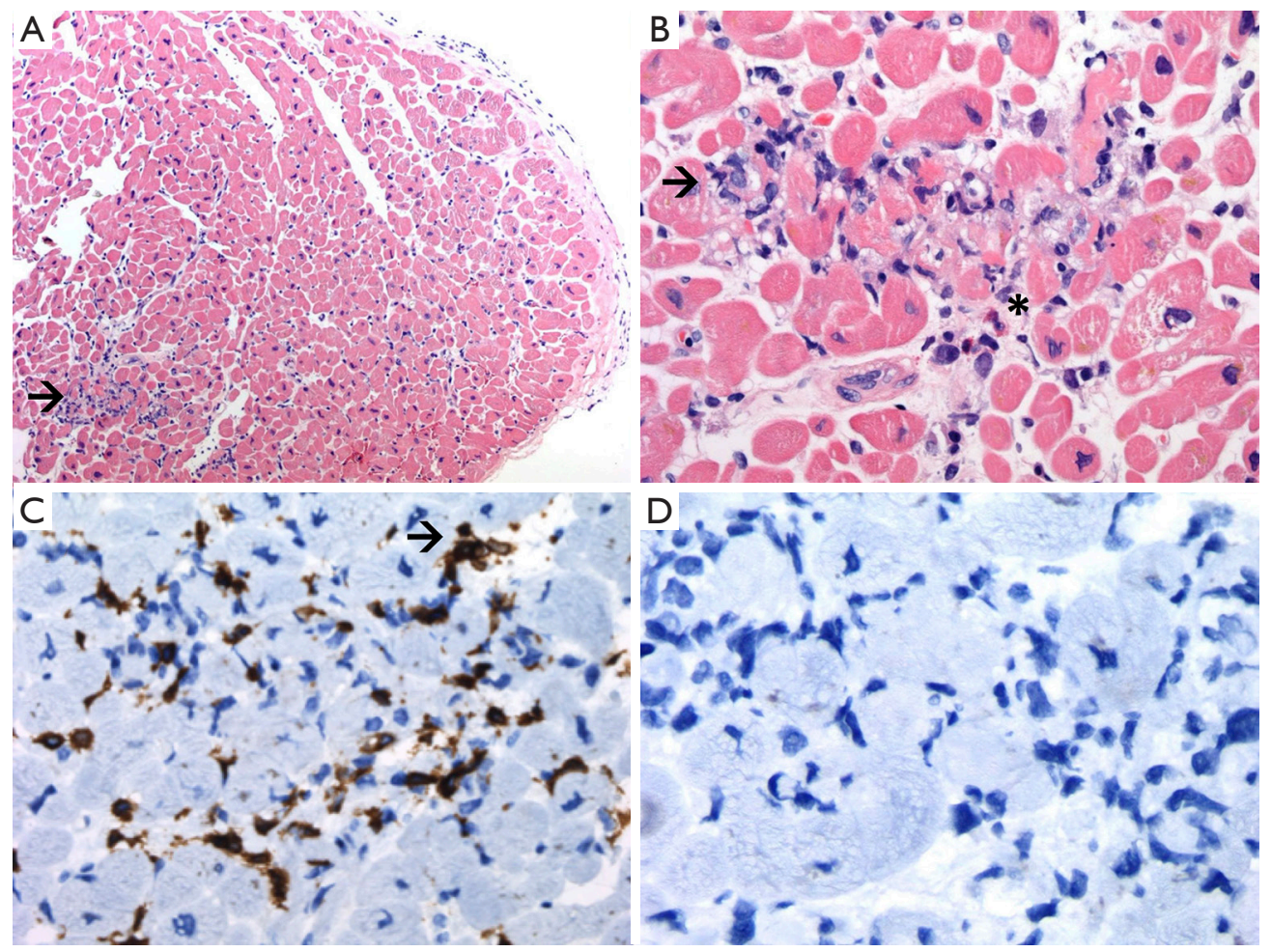

Figure $4 \mathrm{LV}$ endomyocardial biopsy. (A) HE staining $(\times 100)$ displaying endomyocardial and myocardial lymphocytic infiltrates. Arrow: lymphocitic infiltrate. (B) Myocardial biopsy shows lymphocytic infiltrate with eosinophils and myocyte damage (HE, $\times 400)$. Arrow: lymphocitic infiltrate. * indicates eosinophil infiltrate. (C) Positive immunostain CD3 ×400 (polyclonal anti-CD3; Dako omnis). Arrow: CD3+ T lymphocyte. (D) Negative immunostain CD20 ×400 (L26; Dako Omnis). LV, left ventricular; HE, hematoxylin-eosin.

initiated, but symptoms rapidly progressed to severe ptosis, dysphagia and shortness of breath. Despite the treatment received, the patient remained critically ill due to profound cardiogenic shock and respiratory failure. Given the poor short-term prognosis of the underlying disease, aggressive interventions such as intubation and extracorporeal membrane oxygenation (ECMO) implantation were not considered. Comfort measures were adopted and the patient passed away on the $10^{\text {th }}$ day of admission.

All procedures performed in studies involving human participants were in accordance with the ethical standards of the institutional and/or national research committee(s) and with the Helsinki Declaration (as revised in 2013). Written informed consent was obtained from the patient.

\section{Discussion}

ICIs have changed clinical practice in oncology over the past decade. The frequency of irAEs varies according to the specific class of compounds and patient population and are generally manageable. Grade 3-4 toxicities have been reported in $10-27 \%$ of patients receiving anti-CTLA-4, and in $7-20 \%$ of patients receiving anti-PD-1/anti-PD-L1 agents (5). Cardiac events from ICI therapy are not as rare as thought. Mahmood et al. (4) found that $1.14 \%$ patients treated with ICIs developed acute myocarditis and nearly one-half of these cases experienced a mayor adverse cardiovascular event (MACE) (complete heart block, cardiogenic shock, cardiovascular death or cardiovascular arrest), more frequent with the combination than with monotherapy. Elevated serum troponin identified patients at high risk for MACE and a high-dose corticosteroids treatment regimen was associated with a lower troponin level and lower rate of MACE. D'Souza et al. (6) found higher absolute risks, with 1-year absolute risk of cardiac events ranging from $6.6 \%$ to $9.7 \%$ in patients with lung cancer or malignant melanoma. Cardiac events were defined in this study as admission with arrhythmia, myocarditis, pericarditis, heart failure or cardiovascular death. Numerous risk factors for developing ICI-mediated cardiotoxic effects 


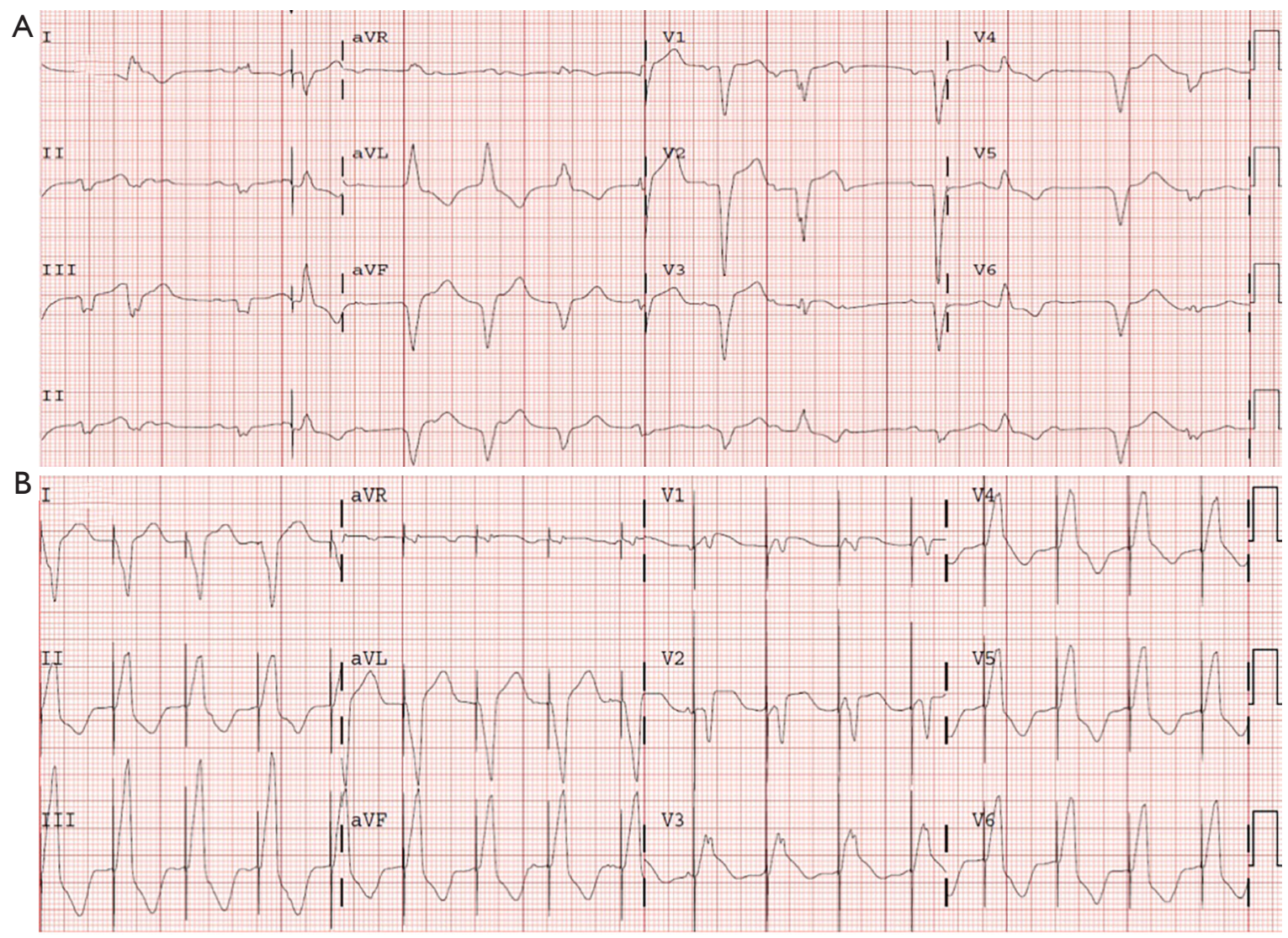

Figure 5 Serial ECGs. (A) ECG exhibits a rapid and polymorphic ventricular rhythm, where ventricular complexes show significant difference in QRS duration, morphology and axis. (B) ECG demonstrating that ventricular pacing successfully suppresses previous ventricular rhythm. ECG, electrocardiogram.

have been reported, and include previous myocardial infarction, heart failure, myocarditis, anthracycline chemotherapy or previous cancer therapy-induced LV dysfunction (7).

Considering the risk for a rapid progression, even mildly elevated cardiac biomarkers should warrant consideration of ICI-induced myocarditis. Cardiac magnetic resonance is the preferred non-invasive test for diagnosis and should be contemplated in stable patients in whom even subtle myocarditis is presumed (8). This could have been the scenario of our patient when she first presented to the emergency department with normal echocardiogram and ECG but a mild increase of troponin levels. At the time of discharge, given the absence of myocarditis-associated symptoms, pleural effusion was assumed to be secondary to baseline illness and troponin elevation attributed to the acute procedure itself. Furthermore, treatment with high-dose corticosteroids should be rapidly initiated if a significant irAE is suspected, and escalation to other immunosuppressive therapies (infliximab, mycophenolate mofetil or anti-thymocyte globulin in case of myocarditis, or intravenous immunoglobulin or plasmapheresis in case of severe neurologic toxicity) may be required if symptoms do not respond promptly $(5,8)$.

Suspicion of ICI-induced myocarditis in our patient was raised early after readmission and intravenous methylprednisolone infusion was quickly started. Other etiologies of complete atrioventricular block, such as radiotherapy-induced conduction system disease and cardiac sarcoidosis, were considered in this patient but ruled out according to the clinical course and tests results. The patient was also assumed to have de novo myasthenia gravis given the absence of previous neurologic symptoms.

Thymic epithelial tumors are more often associated with ICI-related myocarditis (9), as described in the nonrandomized phase II trial that evaluated pembrolizumab 
in platinum-based chemotherapy refractory patients, with high rates of grade $\geq 3$ irAEs in patients with thymoma $(71 \%)$, including myocarditis, myasthenia gravis and hepatitis (10). Concurrence of myocarditis and myasthenia gravis after immune therapy with pembrolizumab in a metastatic thymoma patient has already been described (11). Our patient received aggressive medical treatment with high-dose corticosteroids, infliximab and intravenous immunoglobulin because options for the management of advanced heart failure and myasthenia gravis were limited in the setting of active malignancy.

In brief, myocarditis is a rare but potentially fatal adverse event related to ICI therapy and must be considered in all patients presenting with cardiovascular symptoms and ECG or troponin test abnormalities. As the rate of MACE events in these patients is high, treatment should be instituted promptly with high-dose corticosteroids and escalated if necessary. Consultation with an oncologist is crucial for proper management.

\section{Acknowledgments}

Funding: None.

\section{Footnote}

Reporting Checklist: The authors have completed the CARE reporting checklist. Available at https://dx.doi. org/10.21037/cdt-21-147

Conflicts of Interest: All authors have completed the ICMJE uniform disclosure form (available at https://dx.doi. org/10.21037/cdt-21-147). The authors have no conflicts of interest to declare.

Ethical Statement: The authors are accountable for all aspects of the work in ensuring that questions related to the accuracy or integrity of any part of the work are appropriately investigated and resolved. All procedures performed in studies involving human participants were in accordance with the ethical standards of the institutional and/or national research committee(s) and with the Helsinki Declaration (as revised in 2013). Written informed consent was obtained from the patient for publication of this case report and accompanying images. A copy of the written consent is available for review by the editorial office of this journal.
Open Access Statement: This is an Open Access article distributed in accordance with the Creative Commons Attribution-NonCommercial-NoDerivs 4.0 International License (CC BY-NC-ND 4.0), which permits the noncommercial replication and distribution of the article with the strict proviso that no changes or edits are made and the original work is properly cited (including links to both the formal publication through the relevant DOI and the license). See: https://creativecommons.org/licenses/by-nc$\mathrm{nd} / 4.0 \%$

\section{References}

1. Robert C. A decade of immune-checkpoint inhibitors in cancer therapy. Nat Commun 2020;11:3801.

2. Brahmer JR, Lacchetti C, Schneider BJ, et al. Management of immune-related adverse events in patients treated with immune checkpoint inhibitor therapy: American Society of Clinical Oncology Clinical Practice Guideline. J Clin Oncol 2018;36:1714-68.

3. Majem M, García-Martínez E, Martinez M, et al. SEOM clinical guideline for the management of immunerelated adverse events in patients treated with immune checkpoint inhibitors (2019). Clin Transl Oncol 2020;22:213-22.

4. Mahmood SS, Fradley MG, Cohen JV, et al. Myocarditis in patients treated with immune checkpoint inhibitors. J Am Coll Cardiol 2018;71:1755-64.

5. Haanen JBAG, Carbonnel F, Robert C, et al. Management of toxicities from immunotherapy: ESMO Clinical Practice Guidelines for diagnosis, treatment and followup. Ann Oncol 2017;28:iv119-42.

6. D'Souza M, Nielsen D, Svane IM, et al. The risk of cardiac events in patients receiving immune checkpoint inhibitors: a nationwide Danish study. Eur Heart J 2021;42:1621-31.

7. Lyon AR, Yousaf N, Battisti NML, et al. Immune checkpoint inhibitors and cardiovascular toxicity. Lancet Oncol 2018;19:e447-58.

8. Palaskas N, Lopez-Mattei J, Durand JB, et al. Immune checkpoint inhibitor myocarditis: pathophysiological characteristics, diagnosis, and treatment. J Am Heart Assoc 2020;9:e13757.

9. De Velasco G, Je Y, Bossé D, et al. Comprehensive metaanalysis of key immune-related adverse events from CTLA-4 and PD-1/PD-L1 inhibitors in cancer patients. Cancer Immunol Res 2017;5:312-8. 
10. Cho J, Kim HS, Ku BM, et al. Pembrolizumab for patients with refractory or relapsed thymic epithelial tumor: an open-label phase II trial. J Clin Oncol 2019;37:2162-70.

Cite this article as: Portolés Hernández A, Blanco Clemente M, Escribano García D, Velasco Calvo R, Núñez García B, Oteo Domínguez JF, Salas Antón C, Méndez García M, Segovia Cubero J, Domínguez F. Checkpoint inhibitor-induced fulminant myocarditis, complete atrioventricular block and myasthenia gravis-a case report. Cardiovasc Diagn Ther 2021;11(4):1013-1019. doi: 10.21037/cdt-21-147
11. Szuchan C, Elson L, Alley E, et al. Checkpoint inhibitorinduced myocarditis and myasthenia gravis in a recurrent/ metastatic thymic carcinoma patient: a case report. Eur Heart J Case Rep 2020;4:1-8. 